\title{
Heli4: A Parallel Robot for Scara Motions with a Very Compact Traveling Plate and a Symmetrical Design
}

\author{
Sébastien $\operatorname{Krut}^{(1)}$, Olivier Company ${ }^{(1)}$, Vincent Nabat ${ }^{(1)(2)}$ and François Pierrot ${ }^{(1)}$ \\ (1) LIRMM \\ (2) Fundación FATRONIK \\ (UMR 5506 CNRS - UM2)161 rue Ada \\ 34392 Montpellier CEDEX 5, France \\ Pol. Ibaitarte 1, Apdo. de Correos 160 \\ 20870 Elgoibar (Guipúzcoa), Spain
}

$<$ krut, company,nabat,pierrot>@lirmm.fr

\begin{abstract}
This paper introduces Heli4 a new 4 degree-offreedom parallel robot. It is inspired by the Delta architecture, but was designed to overcome its limitations, using an articulated traveling plate. Unlike most articulated traveling plates, Heli4's traveling plate is very compact. Among other positive aspects is its symmetrical design. This paper gives the geometrical models, and particularly the forward position relationship which can be obtained in a closed form. In a third part, a detailed study of the robot singularities is made by taking into account the not-soclassic internal singularities.
\end{abstract}

Keywords - PKM (Parallel Kinematic Machines), articulated traveling plate, Scara motions, internal singularities, closed-form geometrical models.

\section{INTRODUCTION}

The idea of parallel mechanisms resorting to a non-rigid moving platform (which includes passive joints) and dedicated to Scara motions has been introduced recently and a few academic prototypes have already demonstrated the effectiveness of this principle [1-4]. Indeed, the 4 dof (degrees of freedom) of Scara motions are well adapted to pick-andplace tasks: 3 translations to carry an object from one point to another, plus one rotation for the orientation, around a given axis in world coordinates. Robots inspired from Delta [5] architecture encountered a real commercial success achieving this task, because of their high dynamics. This is due to the lightweight (actuators are fixed on the base) parallel (having closed kinematics chains) design. However, the RUPUR kinematic chain $(R$ stands for Revolute, $U$ for Universal, $P$ for Prismatic, bold letter stands for actuated joint), that transmits the rotational motion using a telescopic leg from a revolute actuator fixed on the frame to the end-effector, may become a weak point (this is particularly true for Delta with huge workspace or, even more, with linear Delta). Most of recent researches in that field have proposed different designs for obtaining Scara motions; some of them are parallel mechanisms, like Kanuk [6], some others have non-fullyparallel designs [7]. Other four-dof parallel mechanisms have been studied in the past, but they are dedicated to different applications such as Koevermans' flight simulator [8] and Reboulet's four-dof wrist [9]. Among recent work on 4-dof PKM dedicated to pick-and-place, we have focused our efforts on various solutions, such as $\mathrm{H} 4$ [1] (introducing the concept of articulated traveling plate), I4 [2] (with a Translation-toRotation transformation device to obtain a symmetrical design and very simple models), Par4 [3] (with a $\pi$ joint, a $R R R R$ planar parallelogram, to mimic the I4's P joint, but with better reliability when subject to high loads) and Dual4 [4] (having an unlimited rotation capability). Not only did we study those machines in detail but we also tested them intensively. From those studies and tests, we have learned that each previous solution offered some advantages, but we also realized how important the simplicity of design (for reliability) and the compactness of the traveling plate (for performances) are. We have then decided to search for a simpler design with a smaller traveling plate.

In this paper, such a new solution is described, and the way to achieve the desired rotation is discussed. Then, geometrical models are derived. A nice feature of this robot is that the forward geometrical model can be written in a closed form. Afterward, a kinematic modeling able to testify to all the singularities of the robot is established: this is based on a detailed modeling of the so-called "spatial parallelograms" which are described here for what they really are (two $S S$ chains). From all this, the geometrical condition that must be validated in order to get the desired motions, shows up.

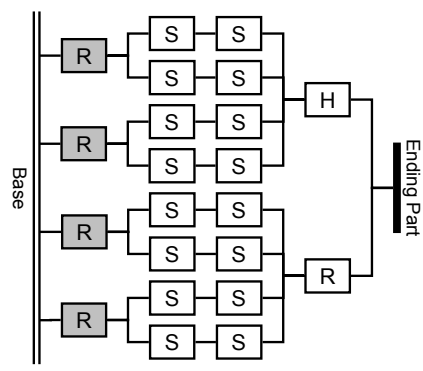

Figure 1. Heli4's joint-and-loop graph; lines represent parts, boxes represent joints (gray boxes mean actuated joints); S stands for Spherical, R for Revolute and $\mathrm{H}$ for Helical.

\section{DESCRIPTION OF THE PROTOTYPE}

The main difference with the FlexPicker (which gave its forearms and parallelograms, see Figure 2, left hand side) is, of course, the use of 4 parallelograms instead of 3. More important, instead of being rigid, the moving platform is articulated and does not require the RUPUR kinematic chain transmitting the rotational motion to the effector. It is composed of two different parts (parts \#1 and \#2), each one being linked by two spatial parallelograms to the actuators, and by a screw (see Figure 2, right hand side). When the two parts move closer, the screw rotates, orientating the ending part. This design makes the articulated traveling plate very compact. The whole architecture gives a workspace similar to the FlexPicker's one, a 1-meter radius, 0.2-meter high cylinder, but overcomes the problems due to the effector's 
rotation. The brushless revolute actuators are associated to gear units with very low backlash $\left(<1^{\prime}\right)$. Moving parts are intended to be as light as possible: forearms and parallelograms are carbon fiber parts (from ABB Robotics), while the traveling plate is made of aluminum. The expected performances for this robot are above $100\left[\mathrm{~m} / \mathrm{s}^{2}\right]$ acceleration and $10[\mathrm{~m} / \mathrm{s}]$ velocity (Note: It is too early to guarantee that such performances will be reached, even though our first tests are encouraging).

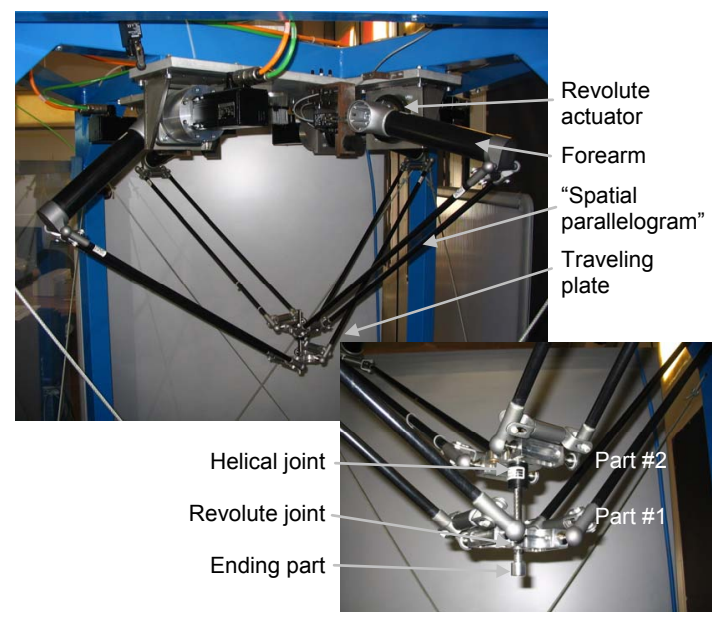

Figure 2. Pictures of the Heli4 prototype; left hand side: view of the whole mechanism; right hand side: zoom on the articulated traveling plate

\section{MODELING}

\section{A. Description of the geometry}

As it is usual for most parallel robots, both relationships, for position and for velocity, are computed using geometrical points $\mathrm{A}_{i j}$ and $\mathrm{B}_{i j}$ defined, using respectively the joint coordinates, and the operational coordinates (see Figure 3 to locate those points). In a practical manner, these models are computed using points $\mathrm{A}_{i}$ and $\mathrm{B}_{i}$, the virtual points located at the center of points $\mathrm{A}_{i 1}$ and $\mathrm{A}_{i 2}$, respectively $\mathrm{B}_{i 1}$ and $\mathrm{B}_{i 2}$. This consideration can be done while assuming that parts \#1 and \#2 of the traveling plate keep the same orientation, when moved in the workspace of the robot (see Figure 2, right hand side). We will see in the next section how to check this hypothesis, or in other words how to make sure that no singularity occurs making the traveling plate lose its constant orientation. Let us now introduce the appropriate notation to do the modeling:

Each geometrical vector $\vec{u}$ (or $\overrightarrow{\mathrm{AB}}$, the vector going from point $\mathrm{A}$ to $\mathrm{B}$ ) will be represented by a column vector $\boldsymbol{u}$ (respectively $\boldsymbol{A B}$ ) expressed in the canonic base $\mathcal{B}=\left(\vec{e}_{x}, \vec{e}_{y}, \vec{e}_{z}\right)$. Moreover, each geometrical point $\mathrm{P}$ will be represented by the column vector $\boldsymbol{P}$ expressed in frame $\mathfrak{R}=\prec \mathrm{O}, \mathcal{B} \succ$ (see Figure 3 ). For example $\boldsymbol{e}_{z}=\left[\begin{array}{lll}0 & 0 & 1\end{array}\right]^{\mathrm{T}}$ is the third canonic vector $\vec{e}_{z}$.

a $\boldsymbol{x}=\left[\begin{array}{llll}x & y & z & \theta\end{array}\right]^{\mathrm{T}}$ is the generalized operational vector. $\boldsymbol{M}=\left[\begin{array}{lll}x & y & z\end{array}\right]^{\mathrm{T}}$ is the column vector associated to $\mathrm{M}$, the tool controlled point, and $\theta$ is the orientation angle of the tool (measured around $\vec{e}_{z}$, starting from $\vec{e}_{x}$ ).

a $\boldsymbol{q}=\left[q_{i}\right]$ is the generalized joint vector. $q_{i}$ are the joint coordinates, $i \in\{1,2,3,4\}$ (angles measured around $\vec{v}_{i}$ starting from $\vec{e}_{z}$ ).

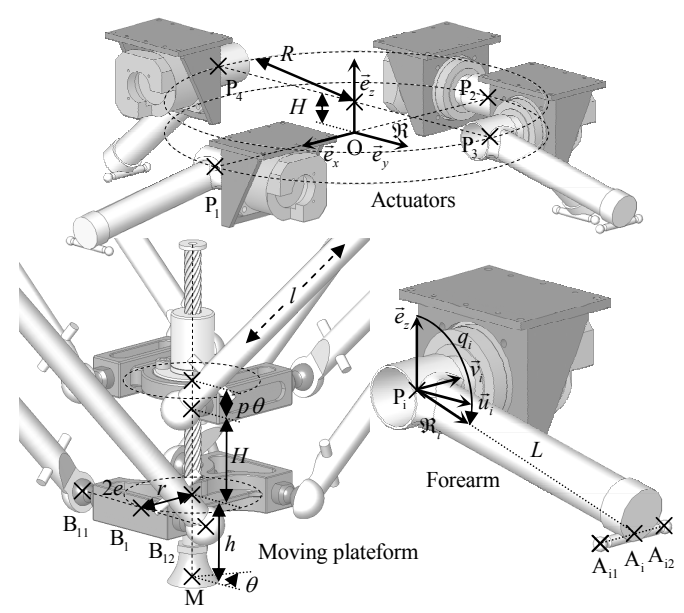

Figure 3. Description of the Heli4's geometry; Top of the figure: geometry of the four actuators; bottom-left: geometry of the articulated traveling plate; bottom-right: geometry of forearm $i$

In the following we will use a cylindrical representation of the geometrical points, characterizing the geometry of the robot, as Heli4 presents a cylindrical symmetry:

口 Vector $\vec{u}_{i}$ is introduced:

$$
\boldsymbol{u}_{i}=\left[\begin{array}{lll}
\cos \left(\alpha_{i}\right) & \sin \left(\alpha_{i}\right) \quad 0
\end{array}\right]^{\mathrm{T}}, i \in\{1,2,3,4\},
$$

where $\alpha_{i}$ are angles measured around $\vec{e}_{z}$ starting from $\vec{e}_{x}$.

口 $\mathrm{P}_{i}, \quad i \in\{1,2,3,4\}$, correspond to the location of the actuators:

$$
\begin{gathered}
\boldsymbol{P}_{i}=R \boldsymbol{u}_{i} \text { for } i \in\{1,2\} \text { and } \\
\boldsymbol{P}_{i}=R \boldsymbol{u}_{i}+H \boldsymbol{e}_{z} \text { for } i \in\{3,4\} \text { (see Figure 3). }
\end{gathered}
$$

$\checkmark$ Points $\mathrm{A}_{i}, \quad i \in\{1,2,3,4\}$ are derived using the Chasle relationship:

$$
\begin{gathered}
\boldsymbol{A}_{i}=\boldsymbol{P}_{i}+\boldsymbol{L}_{i}, i \in\{1,2,3,4\} \text { with: } \\
\boldsymbol{L}_{i}=\left[L \sin \left(q_{i}\right) \cos \left(\alpha_{i}\right) \quad L \sin \left(q_{i}\right) \sin \left(\alpha_{i}\right) \quad L \cos \left(q_{i}\right)\right]^{\mathrm{T}}
\end{gathered}
$$

defined using the joint coordinates ( $L$ is the length of the bars).

口 Points $\mathrm{B}_{i}$ are defined using the operational coordinates:

$$
\boldsymbol{B}_{i}=\boldsymbol{M}+r \boldsymbol{u}_{i}+\left(h_{i}+p_{i} \theta\right) \boldsymbol{e}_{z} \text { for } i \in\{1,2,3,4\}
$$

where $h_{i}$ and $p_{i}$ are defined as follows:

$$
\begin{gathered}
h_{i}=h \text { for } i \in\{1,2\} \text { and } h_{i}=h+H \text { for } i \in\{3,4\} \\
p_{i}=0 \text { for } i \in\{1,2\} \text { and } p_{i}=p \text { for } i \in\{3,4\}
\end{gathered}
$$

$h$ and $H$ are geometrical lengths and $p$ is the pitch of the helical joint (see Figure 3). Numerical values for all the geometrical parameters on the built prototype are listed in Table $1 . \alpha_{i}, i \in\{1,2,3,4\}$ were chosen to give the machine a symmetrical aspect. Lengths of forearms $L$ and single rods $l$ 
were measured on the parts taken from the Flexpicker robot. $H$ was chosen equal to zero for practical reasons. Starting from these definitions, we will see how to derive relationships between $\boldsymbol{x}$ and $\boldsymbol{q}$ in the next section.

TABLE 1

NUMERICAL VALUES FOR THE GEOMETRICAL PARAMETERS OF THE HELI4 PROTOTYPE

\begin{tabular}{|l|l|l|}
\hline \multicolumn{3}{c}{ OF THE HELI4 PROTOTYPE } \\
\hline$\alpha_{1}=0^{\circ}$ & $L=351 \mathrm{~mm}$ & $R=350 \mathrm{~mm}$ \\
\hline$\alpha_{2}=180^{\circ}$ & $l=800 \mathrm{~mm}$ & $r=41 \mathrm{~mm}$ \\
\hline$\alpha_{3}=90^{\circ}$ & $H=0 \mathrm{~mm}$ & $p=50 \mathrm{~mm} /$ round \\
\hline$\alpha_{1}=270^{\circ}$ & $h=41 \mathrm{~mm}$ & \multicolumn{1}{|c}{} \\
\cline { 1 - 2 } & &
\end{tabular}

\section{B. Position relationships}

As it is common for parallel robots having rods which lengths are fixed, the position relationships are derived solving the following equality:

$$
\left\|\boldsymbol{A}_{i} \boldsymbol{B}_{i}\right\|=l, i \in\{1,2,3,4\}
$$

using respectively the operational coordinates for the direct relationship, and the joint coordinates for the inverse relationship.

\section{1) Inverse position relationship}

The resolution is derived as in [10], for robots with rotational actuators, and leads to:

$$
M_{i} \cos \left(q_{i}\right)+N_{i} \sin \left(q_{i}\right)=G_{i}, i \in\{1,2,3,4\}
$$

where: $\quad M_{i}=2 L\left(\boldsymbol{B}_{i} \boldsymbol{P}_{i} \cdot \boldsymbol{e}_{z}\right), N_{i}=2 L\left(\boldsymbol{B}_{i} \boldsymbol{P}_{i} \cdot \boldsymbol{u}_{i}\right)$,

$$
G_{i}=l^{2}+\left\|\boldsymbol{B}_{i} \boldsymbol{P}_{i}\right\|^{2}-L^{2}, \boldsymbol{B}_{i} \boldsymbol{P}_{i}=\boldsymbol{P}_{i}-\boldsymbol{B}_{i} .
$$

( $\boldsymbol{B}_{i}$ is given by relation (6) when knowing the operational parameters).

Making the following change of variable:

$$
t_{i}=\tan \left(q_{i} / 2\right),
$$

leads to a second degree polynomial equation. Once solved, only the root corresponding to the realistic posture is kept, and the joint coordinates can be written as follow:

$$
q_{i}=2 \tan ^{-1}\left(\frac{-b_{i}+\sqrt{b_{i}^{2}-4 a_{i} c_{i}}}{2 a_{i}}\right), i \in\{1,2,3,4\}
$$

with $a_{i}, b_{i}$ et $c_{i}$ the polynomial coefficients:

$$
a_{i}=G_{i}+M_{i}, b_{i}=-2 N_{i} \text { and } c_{i}=G_{i}-M_{i} .
$$

\section{2) Forward position relationship}

In a general manner, it is always preferable to have the forward position relationship written in a closed form. In fact, unlike the Newton's method, the maximum duration of computation can be guaranteed, and there is no trouble with local maximums. Such a model can be derived for the Heli4 robot. The main reason is that operational parameters can be decoupled: $x$ and $y$ parameters can be derived independently of $z$ and $\theta$. Furthermore, $x$ and $y$ can be computed as the intersection of two ellipses.

\section{a) Decoupling operational variables}

Developing relations (9) about the operational parameters leads to:
$(S):\left\{\begin{array}{c}\left(z+p_{i} \theta\right)^{2}+2 a_{i}\left(z+p_{i} \theta\right)+x^{2}+2 b_{i} x+y^{2}+2 c_{i} y+d_{i}=0 \\ i \in\{1,2,3,4\}\end{array}\right.$

where: $\quad a_{i}=\boldsymbol{s}_{i}^{\mathrm{T}} \boldsymbol{e}_{z}, b_{i}=\boldsymbol{s}_{i}^{\mathrm{T}} \boldsymbol{e}_{\boldsymbol{x}}, c_{i}=\boldsymbol{s}_{i}^{\mathrm{T}} \boldsymbol{e}_{\boldsymbol{y}}$,

$$
d_{i}=s_{i}^{2}-l_{i}^{2}, \boldsymbol{s}_{i}=(r-R) \boldsymbol{u}_{i}+h_{i} \boldsymbol{e}_{z}-\boldsymbol{L}_{i} .
$$

( $\boldsymbol{L}_{i}$ is given by relation (5) when knowing the joint parameters).

By subtracting the two first equations of this system, and the two last the following equalities are derived:

$$
\left\{\begin{array}{r}
z=\frac{\left(b_{2}-b_{1}\right)}{\left(a_{1}-a_{2}\right)} x+\frac{\left(c_{2}-c_{1}\right)}{\left(a_{1}-a_{2}\right)} y+\frac{\left(d_{2}-d_{1}\right)}{\left(a_{1}-a_{2}\right)} \\
z+p \theta=\frac{\left(b_{4}-b_{3}\right)}{\left(a_{3}-a_{4}\right)} x+\frac{\left(c_{4}-c_{3}\right)}{\left(a_{3}-a_{4}\right)} y+\frac{\left(d_{4}-d_{3}\right)}{\left(a_{3}-a_{4}\right)}
\end{array}\right.
$$

When merging those results into $1^{\text {st }}$ and $3^{\text {rd }}$ equation of system (16), the following system composed of two quadrics depending only on $x$ and $y$ is derived:

$$
\left(S^{\prime}\right):\left\{\begin{array}{c}
\alpha_{i} x^{2}+\beta_{i} y^{2}+\chi_{i} x y+\delta_{i} x+\varepsilon_{i} y+\phi_{i}=0 \\
i \in\{1,2\}
\end{array}\right.
$$

where $\alpha_{i}, \beta_{i}, \chi_{i}, \delta_{i}, \varepsilon_{i}$ et $\phi_{i}$ depend on the joint coordinates:

$$
\begin{gathered}
\alpha_{i}=\frac{\left(b_{k}-b_{j}\right)^{2}}{\left(a_{j}-a_{k}\right)^{2}}+1, \beta_{i}=\frac{\left(c_{k}-c_{j}\right)^{2}}{\left(a_{j}-a_{k}\right)^{2}}+1, \chi_{i}=\frac{\left(b_{k}-b_{j}\right)\left(c_{k}-c_{j}\right)}{\left(a_{j}-a_{k}\right)^{2}}, \\
\delta_{i}=2 \frac{\left(d_{k}-d_{j}\right)\left(b_{k}-b_{j}\right)}{\left(a_{j}-a_{k}\right)^{2}}+2 a_{j} \frac{\left(b_{k}-b_{j}\right)}{\left(a_{j}-a_{k}\right)}+2 b_{j}, \\
\varepsilon_{i}=2 \frac{\left(d_{k}-d_{j}\right)\left(c_{k}-c_{j}\right)}{\left(a_{j}-a_{k}\right)^{2}}+2 a_{j} \frac{\left(c_{k}-c_{j}\right)}{\left(a_{j}-a_{k}\right)}+2 c_{j}, \\
\phi_{i}=2 \frac{\left(d_{k}-d_{j}\right)^{2}}{\left(a_{j}-a_{k}\right)^{2}}+2 a_{j} \frac{\left(d_{k}-d_{j}\right)}{\left(a_{j}-a_{k}\right)}+d_{j},
\end{gathered}
$$

with $(i, j, k) \in\{(1,1,2),(2,3,4)\}$.

As a conclusion, starting from system (16) which merges the four operational parameters, a simpler system was derived (21) merging only two ( $x$ and $y$ ) operational parameters.

\section{b) Computing $x$ and $y$ as the intersection of 2 ellipses}

Once operational parameters are decoupled, the focus is given to the resolution of parameters $x$ and $y$. They correspond to the intersection of two ellipses as it can be observed on Figure 4. The algebraic solutions of this problem are known [11] and consist in resolving the following $4^{\text {th }}$ degree polynomial equation in $z$ :

$$
P(z)=u_{4} x^{4}+u_{3} x^{3}+u_{2} x^{2}+u_{1} x+u_{0}
$$

obtained with the change of variables:

with: $\quad v_{0}=\alpha_{1} \chi_{2}-\alpha_{2} \chi_{1}, v_{1}=\alpha_{1} \beta_{2}-\alpha_{2} \beta_{1}$,

$$
\begin{gathered}
u_{0}=v_{2} v_{10}-v_{4}^{2}, u_{1}=v_{0} v_{10}+v_{2}\left(v_{7}+v_{9}\right)-2 v_{3} v_{4}, \\
u_{2}=v_{0}\left(v_{7}+v_{9}\right)+v_{2}\left(v_{6}-v_{8}\right)-v_{3}^{2}-2 v_{1} v_{4} \\
u_{3}=v_{0}\left(v_{6}-v_{8}\right)+2 v_{2} v_{5}-2 v_{1} v_{3}, u_{4}=v_{0} v_{5}-v_{1}^{2},
\end{gathered}
$$

$$
\begin{gathered}
v_{2}=\alpha_{1} \delta_{2}-\alpha_{2} \delta_{1}, v_{3}=\alpha_{1} \varepsilon_{2}-\alpha_{2} \varepsilon_{1}, v_{4}=\alpha_{1} \phi_{2}-\alpha_{2} \phi_{1}, \\
v_{5}=\chi_{1} \beta_{2}-\chi_{2} \beta_{1}, v_{6}=\chi_{1} \varepsilon_{2}-\chi_{2} \varepsilon_{1}, v_{7}=\chi_{1} \phi_{2}-\chi_{2} \phi_{1},
\end{gathered}
$$


$v_{8}=\beta_{1} \delta_{2}-\beta_{2} \delta_{1}, v_{9}=\delta_{1} \varepsilon_{2}-\delta_{2} \varepsilon_{1}, v_{10}=\delta_{1} \phi_{2}-\delta_{2} \phi_{1}$.

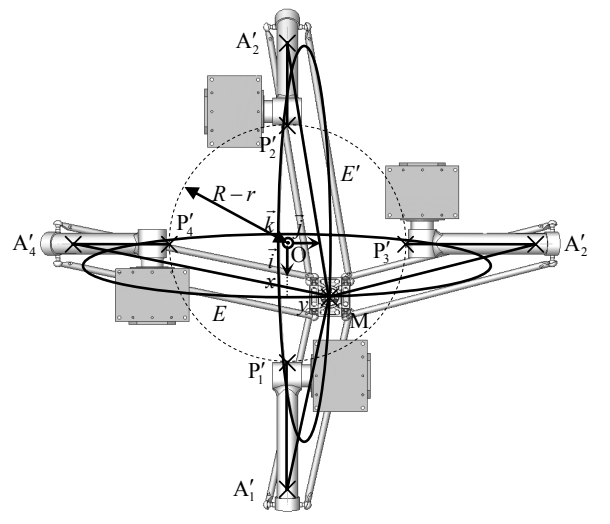

Figure 4. Intersecting ellipses: coordinates $x$ and $y$ correspond to the intersection of the two ellipses $E$ and $E$,

The method proposed by Cardan and Ferrari [12] gives the real roots of polynomial equation (26) in a closed form: it consists in transforming this $4^{\text {th }}$ degree polynomial equation into a $3^{\text {rd }}$ degree polynomial equation. This method presented numerical instabilities and a more robust formulation was chosen [13-15]: it consisted in finding solution either real or complex. In a practical manner, equation (26) has only two real solutions, corresponding to the one observed on Figure 4. So, choosing the proper solution for $x$ consists in keeping only the highest absolute real root (decided from geometrical considerations).

\section{c) Computing all other operational variables}

Once $x$ is determined, $y$ is computed using system (21). Whether than preferring one of the two equations, it is cleverer to subtract both equations in order to eliminate the $y^{2}$ term. It leads to the following expression:

$$
y=\frac{\left(\alpha_{1} \beta_{2}-\alpha_{2} \beta_{1}\right) x^{2}+\left(\alpha_{1} \varepsilon_{2}-\alpha_{2} \varepsilon_{1}\right) x+\left(\alpha_{1} \phi_{2}-\alpha_{2} \phi_{1}\right)}{\left(\alpha_{2} \chi_{1}-\alpha_{1} \chi_{2}\right) x+\left(\alpha_{2} \delta_{1}-\alpha_{1} \delta_{2}\right)}
$$

At last, determining $z$ is obtained using (19), and determining $\theta$ consists in solving system (20):

$$
\theta=\left(\frac{\left(b_{2}-b_{1}\right)}{\left(a_{1}-a_{2}\right)} x+\frac{\left(c_{2}-c_{1}\right)}{\left(a_{1}-a_{2}\right)} y+\frac{\left(d_{2}-d_{1}\right)}{\left(a_{1}-a_{2}\right)}-z\right) / p
$$

In this section, we have seen how to compute both, forward and inverse, position relationships in a close form. In the next section we will derive velocity relationships and focus on singularities.

\section{SINGULARITY ANALYSIS AND VELOCITY RELATIONSHIPS}

In this section we will study the singularities of Heli4. We will focus on the not-so-classic internal singularities. At first we will introduce the Group theory and explain why it is not adapted to the singularity analysis of our robot. The main reason is that parallelograms are composed of spherical joints, and that they can twist (they do not necessary stay planar). Then, using the Grübler method we will list the number of velocity equations and force equations required for the modeling of the whole kinematics of the robot. Simultaneously, we will check that the proposed structure is isostatic. Then, we will recall the singularity analysis method introduced in [16], and apply it to our architecture. We will give the mathematical condition that must be fulfilled to ensure that no internal singularity occurs. Simultaneously velocity relationships will be derived.

\section{A. Preliminary remarks regarding the Group theory}

According to Hervé's notations [17] for displacements subgroups, $\{T\}$ stands for the subgroup of spatial translations and $\{X(\boldsymbol{u})\}$ stands for the subgroup of Schoenflies displacements (or Scara motion), where $\boldsymbol{u}$ is a unitary vector collinear to the rotation's axis. If a closed loop mechanism is composed of two chains producing Schoenflies displacements with $\boldsymbol{v} \neq \boldsymbol{u}$, then:

$$
\{X(\boldsymbol{u})\} \cap\{X(\boldsymbol{v})\}=\{T\}
$$

meaning that such a mechanism will produce only three translations. The case of machines with $R R(R R)_{2} R$ chains (Figure 5-a) is easily handled with such a technique since those chains correspond to Schoenflies subgroup. The case of machines with $\boldsymbol{R}(S S)_{2}$ chains (Figure 5-b) is more complex: each chain provides 5 dof, 3T-2R (3 Translations and 2 Rotations), and does not correspond to a group. Indeed it is possible that the union $(\cup)$ of two $3 \mathrm{~T}-2 \mathrm{R}$ chains generates a 3T-3R motion. The following sub-sections consider precisely this type of $\boldsymbol{R}(S S)_{2}$ chains.

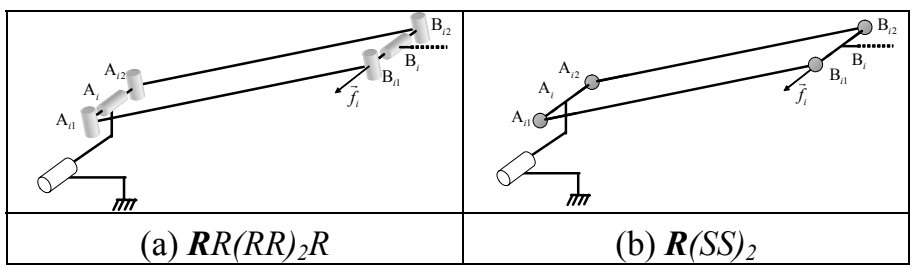

Figure 5. Two ways to do the modeling of parallelograms

\section{B. Grübler analysis}

In this section, based on the Grübler method, we list the number of velocity equations and of force equations required to run the complete analysis of the mechanism. Additionally, we will show that, under the assumption that no singularity occurs, the Heli4 architecture is isostatic.

Using the joint-and-loop graph depicted in Figure 1 the following items can be listed:

व $p=16$ parts

व $n=22$ joints

$\checkmark 5 \mathrm{R}$ joints, $16 \mathrm{~S}$ joints, $1 \mathrm{H}$ joint.

This statement leads to $v$, the number of independent loops:

$$
v=n-p+1=7
$$

Additionally, the total number of dof of the mechanism is:

$$
\sum \mathrm{DoF}=\underbrace{5 \times 1}_{\substack{5 \mathrm{Rjoints} \\ \times 1 \mathrm{DoF}}}+\underbrace{16 \times 3}_{\substack{16 \mathrm{~S} \text { joints } \\ \times 3 \mathrm{DoF}}}+\underbrace{1 \times 1}_{\substack{1 \mathrm{Hjoint} \\ \times 1 \mathrm{DoF}}}=54
$$

Hence, the number of unknown velocities $U_{K}$, of velocity equations $E_{K}$, of unknown forces $U_{S}$, of force equation $E_{S}$, are derived:

$$
U_{K}=\sum \mathrm{DoF}=54
$$




$$
\begin{gathered}
E_{K}=6 v=42 \\
U_{S}=6 n-\sum \mathrm{DoF}=78 \\
E_{S}=6(p-1)=90
\end{gathered}
$$

This statement shows that studying singularities using velocity relations, or the forces relations would involve lots of equations (42 or more). The proposed method, explained in next section, only relies on 8 velocity equations.

The Grübler mobility index $m$ is computed:

$$
m=U_{K}-E_{K}=E_{S}-U_{S}=12 .
$$

As this value is shared between the "kinematic mobility" $m_{C}$ and the "degree of constraint" $m_{S}$ :

$$
m=m_{K}+m_{S}
$$

and as $m_{K}=12$ ( 8 internal motions, each rod being able to rotate around its own axis, plus 4 dof for the whole mechanism) the degree of constraint $m_{S}$ of the mechanism is equal to zero:

$$
m_{S}=0,
$$

meaning that the mechanism is isotatic. We insist on the fact that this relation is true as long as no singularity occurs. This point will be addressed in the following section.

\section{Singularity analysis}

Usually, the study of singularities depends only on the analysis of the standard Jacobian matrices $\boldsymbol{J}_{\boldsymbol{x}}$ and $\boldsymbol{J}_{q}$, satisfying:

$$
\boldsymbol{J}_{q} \dot{\boldsymbol{q}}=\boldsymbol{J}_{x} \dot{\boldsymbol{x}}
$$

where $\dot{\boldsymbol{q}}$ and $\dot{\boldsymbol{x}}$ are respectively the joint velocity vector and the operational velocity vector ${ }^{*}$. But other kinds of singularities can occur [18]. A classification of the different types of singularities is proposed in Table 2. To enlighten them, a deeper analysis needs to be driven. At first, we will recall the basics of the kinematic method developed in order to determine singularities introduced in [16]. Then we will apply the method to Heli4. The geometrical constraints that must be fulfilled to get rid of singularities will be obtained, and it will be checked that, in the whole workspace of the robot, these constraints are satisfied.

The proposed method is well adapted to Heli4 while only single bars separate actuators to the traveling plate. On the one hand, a 4-dof subset made of the actuators can be observed. On the other hand, can be found a 8-dof traveling plate: 3 for positioning, 3 for orientating, and 2 regarding inter-part motilities. Single bars equipped with spherical joints separate both subsets. Each implies that the distance between their extremities is invariant:

$$
\left\|\boldsymbol{l}_{i j}\right\|=l, i \in\{1,2,3,4\}, j \in\{1,2\},
$$

where $\boldsymbol{l}_{i j}$ is the vector joining $\mathrm{A}_{i j}$ to $\mathrm{B}_{i j}\left(\boldsymbol{l}_{i j}=\boldsymbol{A}_{i j}-\boldsymbol{B}_{i j}\right)$.

Deriving this relation leads to the equiprojectivity of velocities of the extremities of each rod:

\footnotetext{
* This notation is well adapted to our study as $\dot{\boldsymbol{q}}=d \boldsymbol{q} / d t$ and $\dot{\boldsymbol{x}}=d \boldsymbol{x} / d t$ (while $\boldsymbol{x}=\left[\begin{array}{llll}x & y & z & \theta\end{array}\right]^{\mathrm{T}}$ ).
}

$$
\boldsymbol{v}_{\mathrm{A}_{i j}}{ }^{\mathrm{T}} \boldsymbol{l}_{i j}=\boldsymbol{v}_{\mathrm{B}_{i j}}{ }^{\mathrm{T}} \boldsymbol{l}_{i j}, i \in\{1,2,3,4\}, j \in\{1,2\},
$$

where $v_{\mathrm{A}_{i j}}$ (respectively $v_{\mathrm{B}_{i j}}$ ) represents the velocity of point $\mathrm{A}_{i j}\left(\mathrm{~B}_{i j}\right)$ relatively to the ground.

TABLE 2

CLASSIFICATION OF THE DIFFERENT TYPES OF SINGULARITIES ON A SIMPLE PLANAR 2-DOF PARALLEL MECHANISM; FOR THE POSTURE REPRESENTED IN THE BOTTOM-RIGHT CELL, THE PARALLELOGRAM CANNOT ENSURE THE TRAVELING PLATE TO REMAIN HORIZONTAL

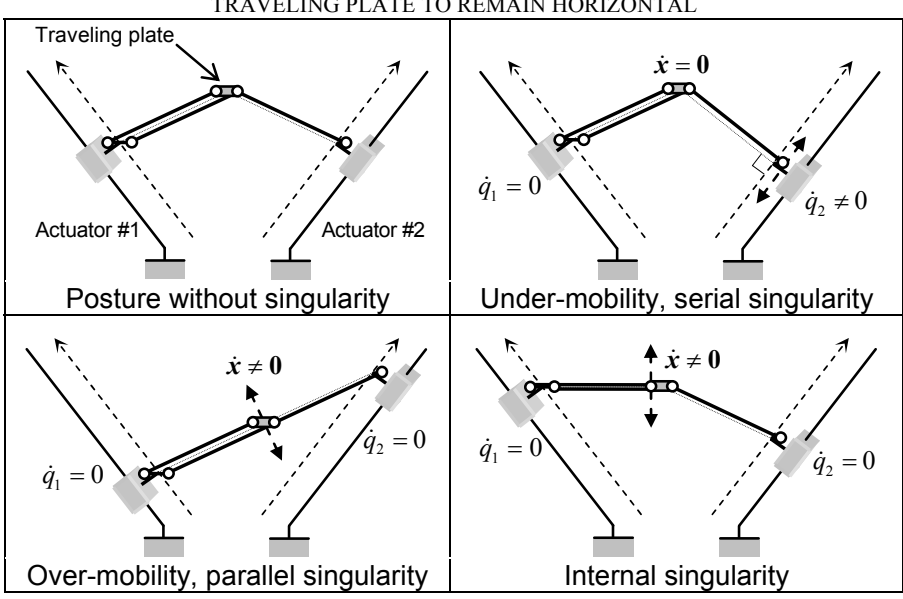

As a consequence, a linear system representative of the whole kinematics of the mechanism can be derived when writing the equiprojectivity relations for the 8 bars:

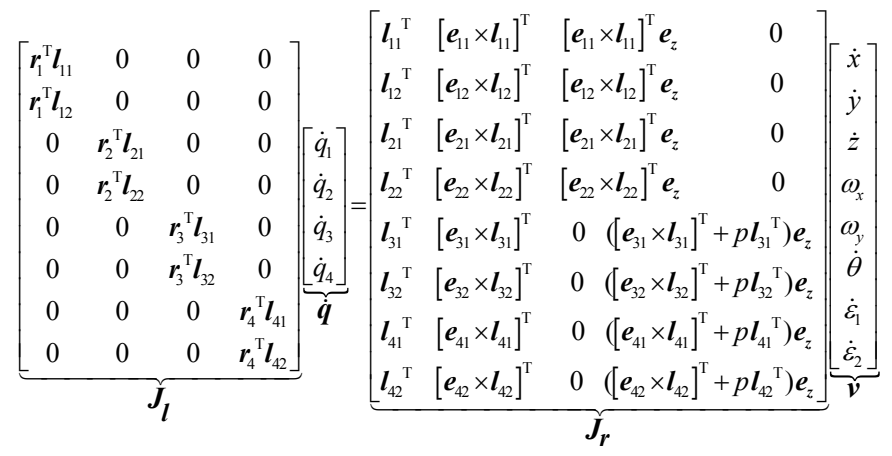

where $\boldsymbol{r}_{i}$ is a vector tangent to the trajectory of points $\mathrm{A}_{i}, \mathrm{~A}_{i 1}$ and $\mathrm{A}_{i 2}$, verifying $\left\|\boldsymbol{r}_{i}\right\|=L, \boldsymbol{e}_{i j}$ is the vector joining $\mathrm{M}$ to $\mathrm{B}_{i j} . \omega_{x}, \omega_{y}$ and $\dot{\theta}$ are the angular velocities of the ending part around $\vec{e}_{x}, \vec{e}_{y}$ and $\vec{e}_{z}$ relatively to the ground, and $\dot{\varepsilon}_{i}$, $i \in\{1,2\}$, are the angular velocities of parts $i$ of the articulated traveling plate relatively to the ending part.

The next step of the method consists in doing elementary operations on this system (which do not affect the rank of the system) to end up with the following system:

$$
\left[\begin{array}{c}
J_{q} \\
\mathbf{0}
\end{array}\right] \dot{\boldsymbol{q}}=\left[\begin{array}{cc}
J_{x} & J_{\text {int }}^{x} \\
\mathbf{0} & J_{\text {int }}
\end{array}\right]\left[\begin{array}{c}
\dot{x} \\
v_{\text {int }}
\end{array}\right],
$$

where $\boldsymbol{J}_{\boldsymbol{i n t}}$ and $\boldsymbol{J}_{\boldsymbol{i n t}}^{\boldsymbol{x}}$ are $4 \times 4$ matrixes and $\boldsymbol{v}_{\boldsymbol{i n t}}$ is a velocity vector. This system has the particularity of being triangular by blocs. In this particular case, this transformation is obtained using the following matrixes: 


$$
\boldsymbol{M}=\left[\begin{array}{cccccccc}
\frac{1}{2} & \frac{1}{2} & 0 & 0 & 0 & 0 & 0 & 0 \\
0 & 0 & \frac{1}{2} & \frac{1}{2} & 0 & 0 & 0 & 0 \\
0 & 0 & 0 & 0 & \frac{1}{2} & \frac{1}{2} & 0 & 0 \\
0 & 0 & 0 & 0 & 0 & 0 & \frac{1}{2} & \frac{1}{2} \\
1 & -1 & 0 & 0 & 0 & 0 & 0 & 0 \\
0 & 0 & 1 & -1 & 0 & 0 & 0 & 0 \\
0 & 0 & 0 & 0 & 1 & -1 & 0 & 0 \\
0 & 0 & 0 & 0 & 0 & 0 & 1 & -1
\end{array}\right], \quad \boldsymbol{P}=\left[\begin{array}{cccccccc}
1 & 0 & 0 & 0 & 0 & 0 & 0 & 0 \\
0 & 1 & 0 & 0 & 0 & 0 & 0 & 0 \\
0 & 0 & 1 & 0 & 0 & 0 & 0 & 0 \\
0 & 0 & 0 & 0 & 1 & 0 & 0 & 0 \\
0 & 0 & 0 & 0 & 0 & 1 & 0 & 0 \\
0 & 0 & 0 & 1 & 0 & 0 & 0 & 0 \\
0 & 0 & 0 & -1 & 0 & 0 & 1 & 0 \\
0 & 0 & 0 & -1 & 0 & 0 & 0 & 1
\end{array}\right] \quad(\operatorname{det}(\boldsymbol{M})=1)
$$

and making the following computation starting from system (49) (this computation does not modify the rank of the system):

$$
\boldsymbol{M} J_{l} \dot{q}=M J_{r} P P^{-1} v
$$

When taking into account the fact that rods $i 1$ and $i 2$ are parallel, computing equation (52) leads to system (50) with the following values:

$$
\begin{aligned}
& \boldsymbol{J}_{\boldsymbol{q}}=\left[\begin{array}{cccc}
\boldsymbol{r}_{1}^{\mathrm{T}} \boldsymbol{l}_{1} & 0 & 0 & 0 \\
0 & \boldsymbol{r}_{2}^{\mathrm{T}} \boldsymbol{l}_{2} & 0 & 0 \\
0 & 0 & \boldsymbol{r}_{3}^{\mathrm{T}} \boldsymbol{l}_{3} & 0 \\
0 & 0 & 0 & \boldsymbol{r}_{4}{ }^{\mathrm{T}} \boldsymbol{l}_{4}
\end{array}\right], \boldsymbol{J}_{\boldsymbol{x}}=\left[\begin{array}{cc}
\boldsymbol{l}_{1}^{T} & 0 \\
\boldsymbol{l}_{2}{ }^{T} & 0 \\
\boldsymbol{l}_{3}{ }^{T} & -p \boldsymbol{l}_{3}^{\mathrm{T}} \boldsymbol{e}_{z} \\
\boldsymbol{l}_{4}{ }^{T} & -p \boldsymbol{l}_{4}^{\mathrm{T}} \boldsymbol{e}_{z}
\end{array}\right],
\end{aligned}
$$

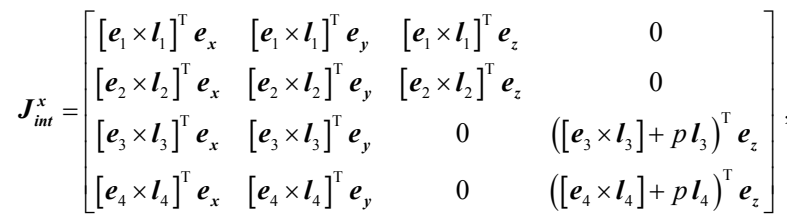

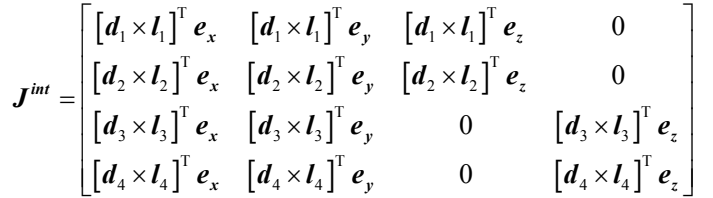

$$
\begin{aligned}
& \text { and: } \quad \boldsymbol{v}_{\boldsymbol{i n t}}=\left[\begin{array}{llll}
\omega_{x} & \omega_{y} & \dot{\theta}+\dot{\varepsilon}_{1} & \dot{\theta}+\dot{\varepsilon}_{2}
\end{array}\right]^{\mathrm{T}} \text {. }
\end{aligned}
$$

( $\boldsymbol{e}_{i}$ is the vector joining $\mathrm{M}$ to $\mathrm{B}_{i}, \boldsymbol{d}_{i}$ the one linking $\mathrm{B}_{i 1}$ to $\mathrm{B}_{i 2}: \boldsymbol{d}_{i}=\boldsymbol{e}_{i 2}-\boldsymbol{e}_{i 1}$.)

$\boldsymbol{J}_{\text {int }}$ will testify to the "internal singularities". In fact, if $\boldsymbol{J}_{\boldsymbol{i n t}}$ is not singular, system (50) implies that:

$$
v_{\text {int }}=\mathbf{0} \text {, }
$$

which means that all parts of the traveling plate always keep the same orientation $\left(\omega_{x}=\omega_{y}=0\right.$ and $\left.\dot{\varepsilon}_{1}=\dot{\varepsilon}_{2}=-\dot{\theta}\right)$ and that coupling between velocities of the different sub-parts exists.

Furthermore, relation:

$$
J_{q} \dot{q}=J_{x} \dot{x}+J_{i n t}^{x} v_{i n t}
$$

derived from (50) falls into the usual velocity relationship (46).

Verifying that $\boldsymbol{J}_{\text {int }}$ is not singular can be done by computing its determinant. It leads to the following relationship:

$$
\left(\left(\left(d_{1} \times l_{1}\right) \times\left(d_{2} \times l_{2}\right)\right) \times\left(\left(d_{3} \times l_{3}\right) \times\left(d_{4} \times l_{4}\right)\right)\right)^{\mathrm{T}} \boldsymbol{e}_{z} \neq 0
$$

By verifying that this relation is always true in the whole workspace, we can guarantee that no "internal singularity" occurs. For other types of singularities, usual Jacobian matrixes need to be studied: $\boldsymbol{J}_{\boldsymbol{q}}$ will enlighten "undermobilities" and $\boldsymbol{J}_{x}$, "over-mobilities" [10]. It is checked that, for this robot, none of these types of singularities are encountered when being inside the workspace of the robot.

\section{CONCLUSION AND FURTHER WORK}

This paper has introduced Heli4, a new 4 Degree-ofFreedom parallel robot. It is inspired by the Delta architecture, but was designed to overcome its limitations, using an articulated traveling plate. Unlike most articulated traveling plates, the one of Heli4 is very compact. Another positive aspect is its symmetrical design. This new robot has been studied in details; the calculation of geometrical models, which are both (forward and inverse) obtained in a close way, was done. Moreover, the study of singularities, using kinematic models, was done resulting in geometrical conditions that must be fulfilled to ensure the absence of singularities, especially the not-so-classic internal singularities.

Up to date, the control of this robot is very simple (linear independent joint control) and will be improved in the future by using, for example, a dynamic controller.

\section{REFERENCES}

[1] Pierrot F., Company O., "H4: a new family of 4-Dof parallel robots", in Proc of IEEE/ASME International Conference on Advanced Intelligent Mechatronics, Atlanta, Georgia, USA, September 19-22, 1999, pp. 508-513.

[2] Krut S., Company O., Benoit M., Ota H. and Pierrot F., "I4: A new parallel mechanism for Scara motions", in Proc. of IEEE ICRA: Int. Conf. on Robotics and Automation, Taipei, Taiwan, September 14-19, 2003.

[3] V. Nabat, M. de la O Rodriguez, O. Company, S. Krut, and F. Pierrot, "Par4: very high speed parallel robot for pick-and-place", in Proc. of IROS 2005: IEEE IRS/RSJ International Conference on Intelligent Robots and Systems, pp. 12021207, Alberta, Canada, August 2-6 2005.

[4] O. Company, F. Pierrot, V. Nabat, M. de la O Rodriguez, "Schoenflies Motion Generator: A New Non-Redundant Parallel Manipulator with Unlimited Rotation Capability", in Proc. of IEEE ICRA: Int. Conf. on Robotics and Automation, Barcelona, Spain, April 18-22, 2005.

[5] R. Clavel, "Une nouvelle structure de manipulateur parallèle pour la robotique légère", APII, 23(6), 1985, pp. 371-386.

[6] Rolland L., "The Manta and the Kanuk: Novel 4 Dof parallel mechanism for industrial handling", in Proc. of ASME Dynamic Systems and Control Division IMECE'99 Conference, Nashville, USA, November 14-19, 1999, vol. 67, pp 831844.

[7] Angeles J., Morozov A., Navarro O., "A novel manipulator architecture for the production of SCARA motions", in Proc. IEEE International Conference on Robotics and Automation, San Francisco, April 24-28, 2000, pp. 2370-2375.

[8] Koevermans W.P. et al., "Design and performance of the four Dof motion system of the NLR research flight simulator", in Proc. of AGARD Conf., No 198, Flight Simulation, La Haye, 20-23 October 1975, pp. 17-1/17-11.

[9] Reboulet C. et al, "Rapport d'avancement projet VAP", thème 7, phase 3 . Rapport de Recherche 7743, CNES/DERA, January 1991.

[10] Merlet J.-P. and Pierrot F., "Modélisation des robots parallèles", in Analyse et modélisation des robots manipulateurs, Lavoisier, ISBN 2-7462-0300-6, 2001, pp. 93-144.

[11] Eberly D., "Intersection of Ellipses", Magic Software Inc., 6006 Meadow Run Court, Chapel Hill, NC 27516, USA.

[12] Candido G., "Le risoluzioni della equazione di quarto grado (Ferrari-EuleroLagrange)", Period. Mat., Vol. 4, No. 21, pp. 88-106, 1941.

[13] Durand E., Solution numérique des équations algébriques, Masson, Paris, 1960.

[14] Démidovitch B. and Maron I., Éléments de calcul numérique, Mir, Moscow, 1979 .

[15] Mineur H., Technique de calcul numérique, Dunod, Paris, 1966.

[16] O. Company, S. Krut and F. Pierrot, "Internal Singularity Analysis of a Class of Lower Mobility Parallel Manipulators with Articulated Travelling Plate", IEEE Transactions on Robotics, 2005.

[17] Hervé J.M., "The Lie group of rigid body displacements, a fundamental tool for mechanism design", Mechanism and Machine Theory, Vol. 34, 1999, pp. 719730 .

[18] Zlatanov D., Fenton R.G., and Benhabib B., "Identification and classification of the singular configurations of mechanisms", Mechanism and Machine Theory, Vol. 33, No. 6, pp. 743-760, August 1998. 